\title{
Cardiac Perforation Caused by Cement Embolus after Cement-Augmented Pedicle Screw Instrumentation: A Case of Report
}

\author{
Hyun Jun Kim${ }^{1}$, Eun Soo Park ${ }^{1}$, Sang Ho Lee ${ }^{2}$, Tak Hyuk Oh${ }^{3}$, Chan Hong Park ${ }^{4}$, Yong Hwan Shin \\ ${ }^{1}$ Department of Neurosurgery, Daegu Wooridul Spine Hospital, Daegu, ${ }^{2}$ Department of Neurosurgery, Wooridul Spine Hospiral, Seoul, \\ ${ }^{3}$ Department of Thoracic and Cardiovascular Surgery, Kyungpook National University Hospital,, Daegu, ${ }^{4}$ Department of Anesthesiology \\ and Pain Medicine, Daegu Wooridul Spine Hospital, Daegu, Korea
}

Corresponding Author:

Eun Soo Park, MD

Department of Neurosurgery, Daegu Wooridul Spine Hospital, 648 Gukchaebosang-ro, Jung-gu, Daegu 41939, Korea

Tel: $+82-53-212-3000$

Fax: +82-53-212-3049

E-mail: seamannn@naver.com

Received: March 12, 2020

Revised: April 13, 2020

Accepted: April 20, 2020
Cement-augmented pedicle screw instrumentation (CAPSI) is a widely used surgical technique in posterior spondylodesis of osteoporotic patients. Complications related to cement leakage after CAPSI was rarely reported compare to that of vertebroplasty, kyphoplasty and most of them are asymptomatic. Herein the authors report the case of a 67-year-old female who experienced cardiac perforation by string shape cement embolus after CAPSI. She underwent the foreign body removal operation through the $5^{\text {th }}$ intercostal thoracotomy and she was well recovered consequently. Surgeons should be aware of this complication entity when encounter postoperative chest pain to avoid fatal results. The authors also reviewed the case reports for cardiac perforation by cement embolus and preventive modalities for the cement leakage.

Key Words: Cardiac perforation by cement embolus, Cement leakage, Cementaugmented pedicle screw, Complications related to cement leakage, Osteoporosis, Vertebroplasty

\section{INTRODUCTION}

As the proportion of elderly individuals in the population increases, the demand for surgical management for osteoporotic patients with poor bone quality has also increased. Pedicle screw insertion in osteoporotic vertebra has become a prevalent challenge. To overcome this issue, cement augmentation of pedicle screw insertion (CAPSI) has been proposed as a feasible surgical technique. Cement augmentation not only facilitates optimal fixation strength but also enhances the density of the instrumented vertebral body, thereby preventing further subsidence of the vertebral body and kyphotic deformity progression ${ }^{9}$. However, there are several complications related to cement insertion such as cement leakage through a nearby venous system. The incidence of pulmonary cement embolism following vertebroplasty or kyphoplasty was reported to be $3.5-23 \%$ and most cases are asymptomatic ${ }^{17)}$. Unlike the complications from cement leakage into the basivertebral veins, leakage into segmental veins was reported to be related to more systemic and severe complications like cardiac perforation ${ }^{31)}$. Herein, we report a rare case of cardiac perforation by a string shaped cement embolus after CAPSI.

\section{CASE REPORT}

\section{History and Examination}

A 67-year-old woman visited the outpatient clinic due to persistent severe back pain and left sciatica with a pain intensity of 8 on the visual analog scale (VAS) over a period of one year. She had a 30-minute walking limit due to neurogenic claudication. Suspecting spinal stenosis, we performed simple tomography (X-ray) on the lumbar region and there was an obvious grade one degenerative spondylolisthesis with dynamic instability on L4-5 (Fig. 1(A). Magnetic resonance imaging (MRI) and computed tomography (CT) were performed for further evaluation and showed spinal stenosis on L3-4-5 with left central intervertebral disc extrusion on L3-4 and vacuum discs on L3-4-5-S1 (Fig. 1(B), C), (D). We decided to manage her conservatively initially and prescribed oral analgesics and physical therapies. She also underwent left L4-5 root blocks with epi- 

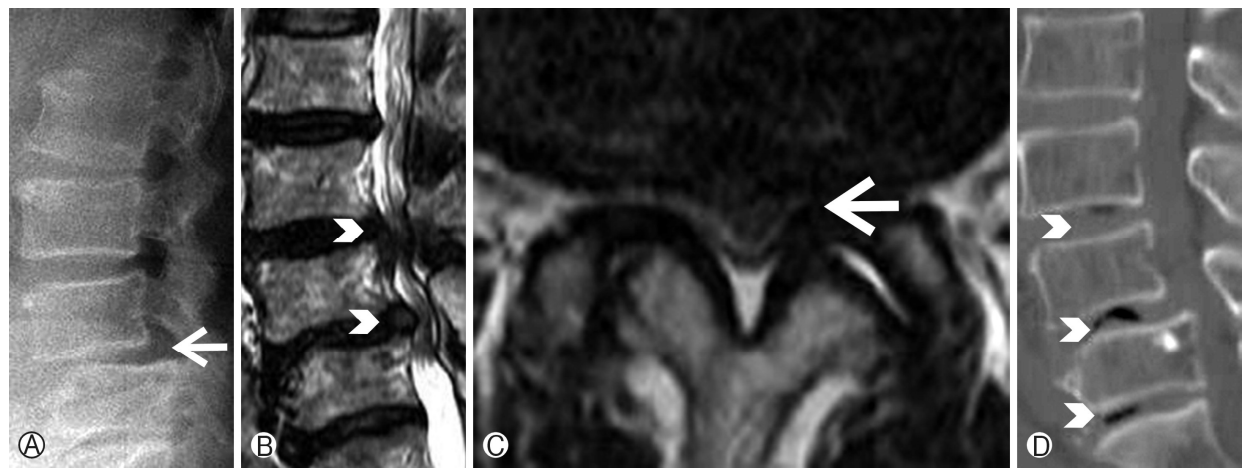

Fig. 1. (A) flexion lumbar lateral simple tomograph shows grade I spondylolisthesis with dynamic instability on L4-5 (white arrow), (B) T2 sequence sagittal magnetic resonance image (MRI) shows spinal stenosis on L3-4-5 (white arrowheads), (C) T2 axial MRI shows concomitant left central disc extrusion with downward migration on L3-4 (white arrow), (D) Sagittal computed tomography shows multiple vacuum intervertebral discs on L3-4-5-S1 (white arrowheads).

dural blocks twice. Her pain was relieved and she could walk for more than 2 hours. However, her pain relapsed after 7 months and persisted after additional epidural blocks. Therefore, we decided to perform unilateral laminectomy and bilateral decompression with discectomy on L3-4 and anterior lumbar interbody fusion on L4-5. On preoperative evaluation, osteoporosis was confirmed by a T-score of -2.5 on bone densitometry.

\section{Operation}

After inserting an interbody cage at L4-5 with an anterior approach, the patient was placed in the prone position for posterior decompression on L3-4 and percutaneous posterior fixation on L4-5. Approximately $6 \mathrm{~mm}$ of $\mathrm{L} 4$ vertebral body reduction was needed for an optimal lumbar lordosis and to decompress L4 exiting roots bilaterally. To overcome her weak bony structure due to osteoporosis and gain additional strength, bone cement augmentation was performed on each pedicle of the $L 4$ vertebral body before the screw insertion. During the cement augmentation, cement leakage was visible through the right segmental vertebral vein and cement insertion was halted immediately (Fig. 2). Consequently $2 \mathrm{cc}$ of cement augmentation was performed on the left side and $1.5 \mathrm{cc}$ of cement augmentation was performed on the right side. There was no obvious unstable hemodynamic change during the entire operation.

\section{Postoperative Course}

On postoperative day (POD) 1, her left sciatica improved to VAS 3 and she could walk without any assistance. On POD 2 , she reported chest wall pain on the right side. A chest $X$-ray showed an asymmetric pleural effusion on the right lung field and a string shaped cement embolus was visible on the right cardiac silhouette (Fig. 3(A)). Since her vital signs were stable, we presumed her chest pain originated from the pleural effusion due to fluid overloading and therefore treated her conservatively for 2 days with diuretics and oral analgesics. On POD 4, her
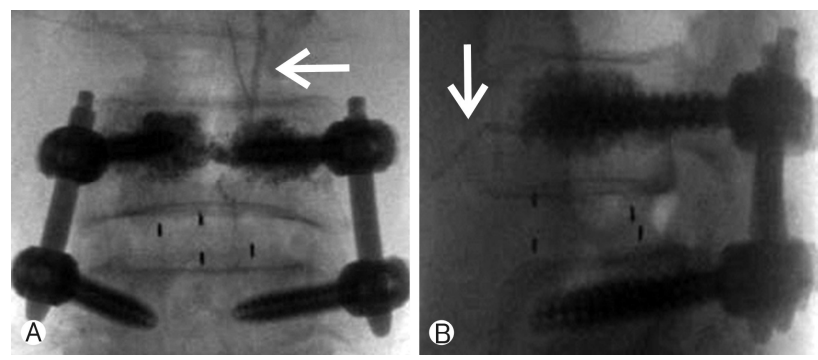

Fig. 2. (A) Anterioposterior and (B) sagittal fluoroscopic images show string shaped cement leakage through the right paravertebral segmental vein (white arrow).
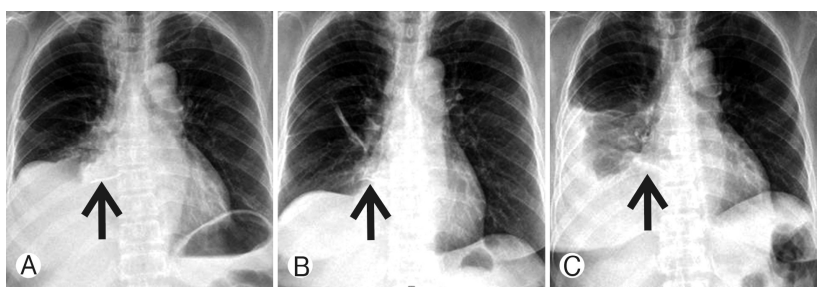

Fig. 3. (A) Posteroanterior chest $X$-ray on postoperative day (POD) 2 shows asymmetric pleural effusion on the right lung field and string shaped cement embolus on the right cardiac silhouette (Black arrow), B After the conservative care pleural effusion subsided on POD4, the cement embolus became more vivid (black arrow), C pleural effusion was aggravated again on POD5.

symptoms and pleural effusion improved (Fig. 3(B)). On POD 5 , her right chest wall pain was aggravated and the pleural effusion had relapsed on the chest X-ray (Fig. 3C). Although there was no hemodynamic instability, she was transferred to a tertiary hospital for further evaluation. At the emergency department, she underwent chest CT, which revealed perforation of the right atrium by a string shaped cement embolus (Fig. 4). She was transferred to the Department of Thoracic 


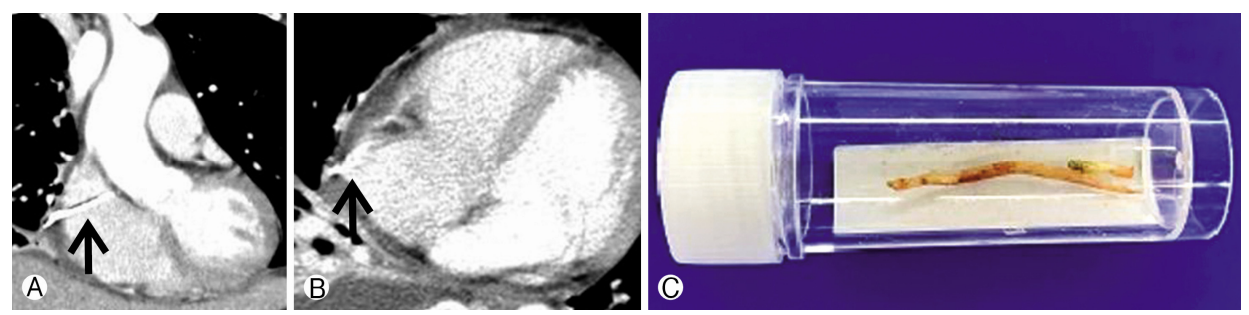

Fig. 4. A, B Chest computed tomography shows the perforation of the right atrium by a string shaped cement embolus (black arrow), (C) A cement embolus with a length of $4.5 \mathrm{~cm}$ was extracted through an intercostal thoracotomy.

and Cardiovascular Surgery and underwent a foreign body removal operation through a $5^{\text {th }}$ intercostal thoracotomy. Under the operation field, a string shaped cement embolus with a length of $4.5 \mathrm{~cm}$ was found to have perforated the pericardium and right atrium and was gently pulled out after placing purse string sutures around it (Fig. 4(C). Fortunately, there was no hemodynamic instability during and after the operation. She was discharged from the tertiary hospital after a week of postoperative care. Two months after the lumbar operation, her radiculopathy and back pain had improved and there was no more chest wall pain.

\section{DISCUSSION}

To study the incidence of cardiac perforation, we performed a PubMed search with the keywords "cement", "cardiac perforation", and "polymethylmethacrylate (PMMA)". Twenty two case reports of cardiac perforation by a string shaped cement embolus were found and these are listed in Table $1^{1,4-7,10-12,15,16,18-27,29,30)}$. Fifteen (68.2\%) of the reported patients underwent vertebroplasty and the rest underwent kyphoplasty $(6,27.3 \%)$. CAPSI was reported for only one patient $(4.5 \%)$ by Andra et al. ${ }^{1)}$. Therefore, this is the second report of cardiac perforation after CAPSI. The operated levels for the 22 patients reported in the literature varied from T8 to S1. Seven (31.8\%) of the operations were at the thoracic level and the others $(15,68.2 \%)$ were limited to the lumbar levels. This shows that an embolus from the lumbar or sacral vertebral levels can also cause cardiac perforation. Most of the patients suffered from chest pain or dyspnea without hemodynamic instability $(19,86.4 \%)$. Interestingly only seven $(31.8 \%)$ patients had these symptoms within a week. The others experienced an insidious onset with a wide range of symptom intervals from 10 days to five years. The insidious onset could be explained by the hypothesis that cement emboli can remained as intra-cardiac emboli until it penetrates or irritates the cardiac wall. Hatzantonis et al. reported a patient with left-sided chest pain, tachycardia and tachypnea 4 weeks after the vertebroplasty ${ }^{12)}$. Echocardiogram revealed intra-cardiac cement emboli without perforation of the right ventricle wall, so they treated patient with enoxaparin at therapeutic dose of $1.5 \mathrm{mg} / \mathrm{kg}$ for 3 months with close follow up exams. They suggest that for patient with other treatable reasons for symptoms, without cardiopulmonary failure could be treated con- servatively.

The insidiouis onset nature of the chest pain could also be the reason why cardiac perforation by cement emboli is underestimated by spine surgeons. Patients are asymptomatic during the postoperative care period, which is usually one to three days. Only two cases (9\%) were reported in a spine-related journal (Farahvar et al., and Tran et al.) ${ }^{10,29)}$. The onset of the patient's chest symptoms was one day after surgery in both reports. The other 20 reports (91\%) were in cardiovascular, chest surgery, or radiology-related journals. Fortunately, two $(9 \%)$ of the patients could be treated with minimally invasive endovascular retrieval; however, most patients were treated with open surgery $(19,86.4 \%)$. One patient could not receive any treatment due to sudden death and cardiac perforation by a cement embolus was found during an autopsy ${ }^{6}$.

Cardiac perforation by a string shaped cement embolus occurred in all patients $(22,100 \%)$. Cement leakage can occur in several types of peri- or intra- vertebral body veins. These include the basivertebral, hemiazygos, azygos, and segmental veins $^{31)}$. The segmental veins are known to connect directly to major vessels like the azygos vein or inferior vena cava (IVC), which connect to the right heart and pulmonary artery ${ }^{31)}$. Therefore, cement leakage to the peri-vertebral segmental veins should be considered the main route for intracardiac cement emboli. We believe the string shape likely forms in the perivertebral segmental veins, which generally have a linear shape. In addition, the right segmental veins are closer to the IVC and shorter than veins on the other side. In our case, there was a linear shaped leakage during the procedure through the L4 right segmental vein (Fig. 2). When leakage in this form is detected, immediate termination of the procedure is essential and thorough reading of the postoperative chest X-ray is required. Even when the leaked cement settles in the segmental vein, it can migrate later to the IVC, heart, or pulmonary artery. To prevent further migration, these fragments can be retrieved preemptively using endovascular snares ${ }^{32}$. A surgical approach should be considered when the cement pieces are too large to be extracted through the femoral catheter window.

There are several suggestions for the prevention of cement emboli. The first is to prevent leakage by thorough fluoroscopic inspection during the procedure. To do so, more vivid visualization of the cement leakage is required by increasing the proportion of barium in PMMA, which can help with early detection. 
Once cement extravasation to the segmental vein is detected, the procedure should be halted because the vein is directly connected to the azygos vein and IVC. Leakage through the segmental veins is detected in 14\% of lateral radiographs and $52 \%$ of anterior-posterior (AP) views ${ }^{31)}$. Since the lateral view is mainly utilized during CAPSI, frequent verification of leakage with the AP view is recommended. Concurrent venography can also be considered before cement injection for selective use embolization agents. However, the use of contrast media for venography may interfere with early detection of cement leakage, and is therefore controversial ${ }^{12)}$. Viscosity is not a measurable value; therefore, despite the ambiguity, toothpaste-like viscosity is generally recommended. Low viscosity can cause early leakage before the hardening of the cement by polymerization of the PMMA.

Sophisticate control of the injection pressure is the key to fill the vertebra sufficiently and to minimize the risk of cement leakage. Broud et al. subdivide pressures should be considered for proper cement injection as follows: extra-vertebral pressure, trabecular cavity pressure, and vertebral cortex pressure $^{2)}$. Since interbody cage of anterior approaches is larger and it covers the wider surface of vertebral end-plate than that of posterior approaches, extra-vertebral pressure and vertebral cortex pressure can be assumed to be higher than that of posterior approaches. Therefore lessor injection pressure with careful inspection during the cement injection should be applied for those cases to minimize the risk of the venous leakage.

Cement leakage can also be prevented by using preventive material or controlling systemic pressure. Gelfoam preinjection embolization was reported to be an effective technique for preventing cement leakage $\mathrm{e}^{3)}$. Bhatia et al. performed gelfoam embolization prior to cement insertion routinely for osteoporotic patients, and reported a $2 \%$ epidural leakage rate ${ }^{3}$. Preoperative placement of an IVC filter could also be considered for severely osteoporotic patients as a preventive step ${ }^{28)}$. El saman et al. insisted that a positive end expiratory pressure of $15 \mathrm{cmH}_{2} \mathrm{O}$ could reduce local cement leakage ${ }^{8)}$. The underlying mechanism is the temporary induction of high pressure in the IVC and segmental vein, which can suppress pressure in the tapped cavity before cement augmentation ${ }^{8)}$.

\section{CONCLUSION}

CAPSI is a generally safe and feasible procedure for osteoporotic patients. However, when a string shaped cement leakage is detected in the peri-vertebral segmental vein and postoperative chest pain develops, cardiac perforation should be considered as a possible cause for the symptom. Prompt evaluation, a high level of suspicion, and immediate surgical or procedural treatment are crucial to treat this disease entity and avoid fatal outcomes.

\section{REFERENCES}

1. Andra M, Baumer H, Mittergradnegger F, Laschitz M, Petek
T, Wandschneider W: Life-threatening cardiac perforation after posterior spondylodesis. The Annals of Thoracic Surgery 104(5): e355-e357, 2017

2. Baroud G, Vant C, Giannitsios D, Bohner M, Steffen T: Effect of vertebral shell on injection pressure and intravertebral pressure in vertebroplasty. Spine 30(1):68-74, 2005

3. Bhatia C, Barzilay Y, Krishna M, Friesem T, Pollock R: Cement leakage in percutaneous vertebroplasty: effect of preinjection gelfoam embolization. Spine 31(8):915-919, 2006

4. Bouchez S, Mauermann E, Philipsen T, Wouters P: 3D echocardiographic diagnosis of right ventricular perforation with polymethylmethacrylate particles after vertebroplasty. Journal of Cardiothoracic and Vascular Anesthesia 31(6):2123-2126, 2017

5. Caynak B, Onan B, Sagbas E, Duran C, Akpinar B: Cardiac tamponade and pulmonary embolism as a complication of percutaneous vertebroplasty. The Annals of Thoracic Surgery 87(1): 299-301, 2009

6. D'Errico S, Niballi S, Bonuccelli D: Fatal cardiac perforation and pulmonary embolism of leaked cement after percutaneous vertebroplasty. Journal of Forensic and Legal Medicine 63:4851, 2019

7. Dreger H, Treskatsch S, Lembcke A, Grubitzsch H, Knebel F, Laule M. Perforation of the right ventricle by bone cement: a rare complication of kyphoplasty. European heart journal. Apr 2013;34(16):1203.

8. El Saman A, Kelm A, Meier S, et al: Intraoperative PEEP-ventilation during PMMA-injection for augmented pedicle screws: improvement of leakage rate in spinal surgery. European Journal of Trauma and Emergency Surgery: Official Publication of the European Trauma Society 39(5):461-468, 2013

9. Elder BD, Lo SF, Holmes C, et al: The biomechanics of pedicle screw augmentation with cement. The Spine Journal: Official Journal of the North American Spine Society 15(6):1432-1445, 2015

10. Farahvar A, Dubensky D, Bakos R: Perforation of the right cardiac ventricular wall by polymethylmethacrylate after lumbar kyphoplasty. Journal of Neurosurgery Spine 11(4):487-491, 2009

11. Gosev I, Nascimben L, Huang PH, et al: Right ventricular perforation and pulmonary embolism with polymethylmethacrylate cement after percutaneous kyphoplasty. Circulation 127(11): 1251-1253, 2013

12. Hatzantonis C, Czyz M, Pyzik R, Boszczyk BM: Intracardiac bone cement embolism as a complication of vertebroplasty: management strategy. European Spine Journal: Official Publication of the European Spine Society, the European Spinal Deformity Society, and the European Section of the Cervical Spine Research Society 26(12):3199-3205, 2017

13. Ishikawa K, Hayashi $\mathrm{H}$, Mori $\mathrm{H}$ : Cardiac perforation caused by cement after percutaneous balloon kyphoplasty. Asian Cardiovascular \& Thoracic Annals 25(3):204-206, 2017

14. Jang JS, Lee SH, Jung SK: Pulmonary embolism of polymethylmethacrylate after percutaneous vertebroplasty: a report of three cases. Spine 27(19):E416-418, 2002

15. Kim SP, Son BS, Lee SK, Kim DH: Cardiac perforation due to intracardiac bone cement after percutaneous vertebroplasty. Journal of Cardiac Surgery 29(4):499-500, 2014

16. Kim SY, Seo JB, Do KH, Lee JS, Song KS, Lim TH: Cardiac perforation caused by acrylic cement: a rare complication of per- 
cutaneous vertebroplasty. AJR. American Journal of Roentgenology 185(5):1245-1247, 2005

17. Krueger A, Bliemel C, Zettl R, Ruchholtz S: Management of pulmonary cement embolism after percutaneous vertebroplasty and kyphoplasty: a systematic review of the literature. European Spine Journal: Official Publication of the European Spine Society, the European Spinal Deformity Society, and the European Section of the Cervical Spine Research Society 18(9):1257-1265, 2009

18. Lee V, Patel R, Meier P, Lawrence D, Roberts N: Conservative management of inferior vena cava cement spike after percutaneous vertebroplasty causes fatal cardiac tamponade. The Journal of Rheumatology 41(1):141-142, 2014

19. Lim SH, Kim H, Kim HK, Baek MJ: Multiple cardiac perforations and pulmonary embolism caused by cement leakage after percutaneous vertebroplasty. European Journal of Cardiothoracic Surgery: Official Journal of the European Association for Cardio-thoracic Surgery 33(3):510-512, 2008

20. Llanos RA, Viana-Tejedor A, Abella HR, Fernandez-Aviles F: Pulmonary and intracardiac cement embolism after a percutaneous vertebroplasty. Clinical Research in Cardiology: Official Journal of the German Cardiac Society 102(5):395-397, 2013

21. Mattis T, Knox M, Mammen L: Intracardiac methylmethacrylate embolism resulting in right atrial wall perforation and pericarditis following percutaneous vertebroplasty. Journal of Vascular and Interventional Radiology: JVIR 23(5):719-720, 2012

22. Moon MH, Jo KH, Kim HW: Cardiac perforation caused by bone cement embolism. Archives of Cardiovascular Diseases 106(6-7):413-414, 2013

23. Park JH, Choo SJ, Park SW: Images in cardiovascular medicine. Acute pericarditis caused by acrylic bone cement after percutaneous vertebroplasty. Circulation 111(6):e98, 2005

24. Prokop A, Hagele M, Pfeilsticker U, Koll S, Chmielnicki M: Pericardial perforation 2.5 years after kyphoplasty. A rare complication after cement extravasation. Der Unfallchirurg 116(1):
80-84, 2013

25. Schoenes B, Bremerich DH, Risteski PS, Thalhammer A, Meininger D: Cardiac perforation after vertebroplasty. Der Anaesthesist 57(2):147-150, 2008

26. Shen C, Liu G, Hu JZ, Yang XH: Cardiac perforation and multiple emboli after percutaneous vertebroplasty. Orthopedics 38(10):e947-950, 2015

27. Son KH, Chung JH, Sun K, Son HS: Cardiac perforation and tricuspid regurgitation as a complication of percutaneous vertebroplasty. European Journal of Cardio-thoracic surgery: Official Journal of the European Association for Cardio-thoracic Surgery 33(3):508-509, 2008

28. Sun HL, Li CD, Yang ZC, et al: Polymethylmethacrylate augmentation of bone cement-injectable cannulated pedicle screws for the treatment of degenerative lumbar diseases with osteoporosis. Beijing da xue xue bao. Yi xue ban=Journal of Peking University. Health Sciences 48(6):1019-1025, 2016

29. Tran I, Gerckens U, Remig J, Zintl G, Textor J: First report of a life-threatening cardiac complication after percutaneous balloon kyphoplasty. Spine 38(5):E316-318, 2013

30. Yang JH, Kim JW, Park HO, Choi JY, Jang IS, Lee CE: Intracardiac foreign body (bone cement) after percutaneous vertebroplasty. The Korean Journal of Thoracic and Cardiovascular Surgery 46(1):72-75, 2013

31. Yeom JS, Kim WJ, Choy WS, Lee CK, Chang BS, Kang JW: Leakage of cement in percutaneous transpedicular vertebroplasty for painful osteoporotic compression fractures. The Journal of Bone and Joint Surgery. British volume. 85(1):83-89, 2003

32. Zhao Y, Liu T, Zheng Y, Wang L, Hao D: Successful percutaneous retrieval of a large pulmonary cement embolus caused by cement leakage during percutaneous vertebroplasty: case report and literature review. Spine 39(26):E1616-1621, 2014 